\title{
Comparing the image potentials for intercalated graphene with a two-dimensional electron gas with and without a gated grating
}

\author{
Godfrey Gumbs, ${ }^{1,3}$ Danhong Huang, ${ }^{2}$ and P. M. Echenique ${ }^{3}$ \\ ${ }^{1}$ Department of Physics and Astronomy, Hunter College of City University of New York, 695 Park Avenue, \\ New York, New York 10065, USA \\ ${ }^{2}$ Air Force Research Laboratory, Space Vehicles Directorate, Kirtland Air Force Base, New Mexico 87117, USA \\ ${ }^{3}$ Departamento de fisica de materials and centro mixto CSIC-UPV and Donostia International Physics Center (DIPC), \\ P. de Manuel Lardizabal, 4, 20018 San Sebastián, Basque Country, Spain
}

(Received 20 August 2008; revised manuscript received 1 December 2008; published 15 January 2009)

\begin{abstract}
We calculate the surface response function and the image potential of a system of layered two-dimensional (2D) electron gas structures. A point charge is placed at a distance away from the surface which lies in the $x y$ plane. These 2D layers are coupled through the Coulomb interaction and there is no interlayer electron hopping. The separation between adjacent layers can be adjusted to investigate the roles which the layer separation and the number of layers play on both the surface response function and the image potential. Specifically, we consider the system composed of graphene layers or the layered 2D electron gas (EG) formed at the interface of a semiconductor heterostructure such as GaAs/AlGaAs. We show that the image potential for graphene is qualitatively the same as for the 2DEG. We examine the way in which the image potential is modified by applying a one-dimensional periodic electrostatic potential (through a gated grating for modulation). The results indicate that the charge screening for graphene is not much different from the 2DEG.
\end{abstract}

DOI: 10.1103/PhysRevB.79.035410

PACS number(s): 32.80.Ee, 33.80.Rv, 24.10.Cn, 73.20.Mf

\section{INTRODUCTION}

It is well established that an external charge at a distance $z_{0}$ outside of a semi-infinite dielectric medium with dielectric constant, $\epsilon$ polarizes the surface charge and becomes attracted to its "image charge" residing below the surface, giving rise to a spatially extended state. ${ }^{1}$ The image-potential states are relevant for excited electrons at surfaces. Due to its long-range $1 / z_{0}$ dependence, the potential supports an infinite number of image states exibiting the well-known Rydberg series form: $E_{n}=-\left(13.6 / 16 n^{2}\right)[(\epsilon-1) /(\epsilon+1)] \mathrm{eV}$, where $n=1,2,3, \ldots$ is the principal quantum number. Since $E_{n} \sim 1 / n^{2}$, the states with higher $n$ have weaker binding energies. This series converges to the vacuum level. The electrons are confined along the surface normal but can move freely parallel to the surface leading to discrete parabolic energy bands. Recently, Höfer et $a .^{2}$ applied two-photon photoemission techniques ${ }^{3}$ to populate the coherent wave packets of image states close to $\mathrm{Cu}(100)$ and $\mathrm{Cu}(111)$ surfaces. The states observed in these experiments had $n \leq 6$ and binding energies of $15-40 \mathrm{meV}$. These surface states collapsed onto the $\mathrm{Cu}$ surface with lifetimes of a few femtoseconds. The states with larger $n$ have longer lifetimes. ${ }^{4}$ For example, for the $\mathrm{Cu}(100)$ surface, the lifetimes of electrons excited to the image-potential states for $n=1,2,3$ are $\tau_{1}$ $\approx 40 \mathrm{fs}, \tau_{2} \approx 110 \mathrm{fs}$, and $\tau_{3} \approx 300 \mathrm{fs}$, respectively. ${ }^{2}$

In this paper, we calculate the image potentials that exist in front of layered two-dimensional (2D) electron systems (ESs), which may be comprised of a $2 \mathrm{D}$ electron gas (EG) or a graphene layer. Of course, an electron near the surface would experience the combined effect of the Coulomb-type attractive image potential and the repulsive surface-barrier potential. ${ }^{4}$ We show that the potential created by the polarization which a charge induces at the surface of a layered 2DES is qualitatively the same for a $2 \mathrm{DEG}$ compared with that of graphene. We also examine how a one-dimensional (1D) periodic potential (through a gated grating for modulation) affects the image potential. The binding energy of image states of intrinsic graphite has been measured by Collins, Andrews, and $\mathrm{Law}^{5}$ using angle-resolved ultraviolet inverse photoemission and by Lehmann et al. ${ }^{6}$ by means of multiphoton photoemission spectroscopy. The crystal structure of graphite consists of carbon atoms arranged in a planar hexagonal network. These layers are stacked following an $A B A B A B A \cdots$ sequence, with half the number of atoms lying directly above those in adjacent planes while half lie above the centers of the hexagons in adjacent layers. The interlayer coupling is weak and is determined by the $2 p_{z}$ (or $\pi$ ) atomic orbitals. The spacing between layers is large compared with the $\mathrm{C}-\mathrm{C}$ bond length. The energy dispersions for the valence and conduction bands for intercalated graphite have been mapped out using angle-resolved inverse photoemission. ${ }^{7}$ We carry out calculations for the image potential of graphene whose energy dispersion for the low-energy states in the valence and conduction bands is linear. The bound states we obtain for the image potential may be observed by angleresolved inverse photoemission, which is an ideal technique for probing bound states directly by measuring their energy and momentum via the energy and momentum of an incident electron and the energy of the emitted photon. ${ }^{8}$ The lifetime of an image-potential state is, in fact, finite and is determined by its possible decay into excitations of the crystal, as well as its leakage into neighboring image states. Granger et al. ${ }^{9}$ predicted that the image potential outside a single-wall carbon nanotube can support a different class of bound states due to the quantizing effect of the centripetal force. This result was later confirmed experimentally. ${ }^{10}$ The image potential for double-wall nanotubes was calculated by Gumbs et $a l .{ }^{11}$ who showed how the inner wall can affect the redistribution of the surface charge and consequently the image states. 


\section{GENERAL FORMULATION OF THE PROBLEM}

A powerful spectroscopic tool which has been utilized for studying dynamical processes at surfaces is electron energyloss spectroscopy. The scattering mechanism of the incident electrons is assumed to be long-ranged and dipolar. Therefore, the electric field due to the external current density can be expressed as $\mathbf{E}_{\text {ext }}=-\vec{\nabla} \phi_{\text {ext }}$. Let the layered system occupy the region $z>0$. Since $\nabla^{2} \phi_{\text {ext }}=0$ for $z<0$, but between the impinging charged particle and the surface at $z=0$, the electrostatic potential in the region $z<0$ and in the vicinity of the surface can be written as a superposition of plane waves. The total potential is the sum of the external and induced potential outside the layered system.

When a 1D periodic electrostatic potential is applied on the surface of the system, the surface response function will be modified from its value in the absence of the modulation. ${ }^{12,13}$ We assume that the periodic potential is along the $x$ direction and that the modulated sheet density can be described by $n_{2 \mathrm{D}}(x)=\sum_{n=-\infty}^{\infty} \rho_{n} \exp (i n G x)$, where the real-value $\rho_{n}$ is the $n$th Fourier component of $n_{2 \mathrm{D}}(x), G$ $=2 \pi / d$ is the reciprocal-lattice vector, and $d$ is the period of the potential. In this case, we have as the potential outside the layered system (see the Appendix for details),

$$
\begin{aligned}
\phi_{<}(\mathbf{r} ; \omega)= & \sum_{n=-\infty}^{\infty} \int \frac{d \mathbf{q}_{\|}}{(2 \pi)^{2}} \\
& \times\left[e^{-q_{n} z}-g_{n}\left(\mathbf{q}_{\|} ; \omega\right) e^{q_{n} z}\right] e^{i q_{x, n} x+i q_{y} y} \text { for } z \lesssim 0,
\end{aligned}
$$

where $q_{n}=\sqrt{q_{x, n}^{2}+q_{y}^{2}}, q_{x, n}=q_{x}+n G$, and $\mathbf{q}_{\|}=\left(q_{x}, q_{y}\right)$ is the 2D wave vector of an induced charge-density wave. We will now calculate the Fourier components of the surface response function $g_{n}\left(\mathbf{q}_{\|} ; \omega\right)$ for a layered 2DES. There is a dielectric medium with dielectric constant $\epsilon$ filling the space between adjacent layers, except for a very thin vacuum region adjacent to the $2 \mathrm{D}$ layer. The electrostatic potential in the vacuum region between layers at $z=z_{\ell}$ and $z=z_{\ell+1}$ is given by $\phi_{\ell}(\mathbf{r}, t)=e^{-i \omega t} \phi_{\ell}(\mathbf{r}, \omega)$, where

$$
\begin{aligned}
\phi_{\ell}(\mathbf{r} ; \omega)= & \sum_{n=-\infty}^{\infty} \int \frac{d \mathbf{q}_{\|}}{(2 \pi)^{2}}\left[A_{\ell}^{(n)} e^{-q_{n}\left(z-z_{\ell}\right)}\right. \\
& \left.+B_{\ell}^{(n)} e^{q_{n}\left(z-z_{\ell}\right)}\right] e^{i q_{x, n} x+i q_{y} y} .
\end{aligned}
$$

Also, at the last interface, we take

$$
\begin{aligned}
\phi_{L+1}(\mathbf{r} ; \omega)= & \sum_{n=-\infty}^{\infty} \int \frac{d \mathbf{q}_{\|}}{(2 \pi)^{2}} e^{i q_{x, n} x+i q_{y} y} t_{L+1} e^{-q_{n}\left(z-z_{L}\right)} \\
& \text { for } z \geq z_{L+1} .
\end{aligned}
$$

Both the potential $\phi$ and its derivative $\epsilon d \phi / d z$ must be continuous at the vacuum-dielectric medium interface. However, the electric field is discontinuous across the 2D charged layer on which the induced electron sheet density is

$$
\sigma_{\ell}^{(n)}\left(q_{n}, \omega\right)=e^{2} \chi^{(0)}\left(q_{n}, \omega\right)\left(A_{\ell}^{(n)}+B_{\ell}^{(n)}\right),
$$

where $\chi^{(0)}\left(q_{n}, \omega\right)$ is the single-particle density-density response function. We emphasize that in this model, we as- sume that the grating period modulates the induced electron density, as described in Eq. (4) in linear-response theory. We use no other parameter to describe the gated grating period. Consequently, we only need to specify the grating potential in our numerical calculations by periodicity $d$. After some straightforward algebra, we obtain

$$
\left[\begin{array}{c}
A_{\ell+1}^{(n)} \\
B_{\ell+1}^{(n)}
\end{array}\right]=\stackrel{\leftrightarrow}{T}_{a_{\ell}}^{(n)}(\alpha)\left[\begin{array}{c}
A_{\ell}^{(n)} \\
B_{\ell}^{(n)}
\end{array}\right]=\mathcal{M}_{\ell}^{(n)}\left[\begin{array}{c}
1 \\
-g\left(q_{n}, \omega\right)
\end{array}\right],
$$

where $g\left(q_{n}, \omega\right) \equiv g_{n}\left(\mathbf{q}_{\|} ; \omega\right)$ depends only on the total wave number

$$
\begin{gathered}
q_{n}=\sqrt{q_{x, n}^{2}+q_{y}^{2}} \\
\mathcal{M}_{\ell}^{(n)}=\overleftrightarrow{T}_{a_{\ell}}^{(n)}(\alpha) \otimes \cdots \otimes \overleftrightarrow{T}_{a_{1}}^{(n)}(\alpha) \otimes \overleftrightarrow{T}_{a_{0}}^{(n)}(\alpha),
\end{gathered}
$$

and

$$
\begin{aligned}
{\left[\overleftrightarrow{T}_{a}^{(n)}(\alpha)\right]_{11}=} & {\left[(1+\epsilon)(\epsilon+1+2 \epsilon \alpha) e^{-q_{n} a}\right.} \\
& \left.+(1-\epsilon)(\epsilon-1-2 \epsilon \alpha) e^{q_{n} a}\right] / 4 \epsilon, \\
{\left[T_{a}^{(n)}(\alpha)\right]_{12}=} & {\left[(1+\epsilon)(\epsilon-1+2 \epsilon \alpha) e^{-q_{n} a}\right.} \\
& \left.+(1-\epsilon)(\epsilon+1-2 \epsilon \alpha) e^{q_{n} a}\right] / 4 \epsilon, \\
{\left[\overleftrightarrow{T}_{a}^{(n)}(\alpha)\right]_{21}=} & {\left[(1-\epsilon)(\epsilon+1+2 \epsilon \alpha) e^{-q_{n} a}\right.} \\
& \left.+(1+\epsilon)(\epsilon-1-2 \epsilon \alpha) e^{q_{n} a}\right] / 4 \epsilon, \\
{\left[\overleftrightarrow{T}_{a}^{(n)}(\alpha)\right]_{22}=} & {\left[(1-\epsilon)(\epsilon-1+2 \epsilon \alpha) e^{-q_{n} a}\right.} \\
& \left.+(1+\epsilon)(\epsilon+1-2 \epsilon \alpha) e^{q_{n} a}\right] / 4 \epsilon .
\end{aligned}
$$

In Eq. (6), $\alpha\left(q_{n}, \omega\right)=\left(e^{2} / 2 \epsilon_{0} \epsilon\right) \chi^{(0)}\left(q_{n}, \omega\right)$ is the polarization function for each $2 \mathrm{D}$ charged layer. When we equate the electrostatic potential just inside the material at the last layer to the electrostatic potential outside, we obtain from Eq. (6), after solving for the surface response function,

$$
\begin{aligned}
& g\left(q_{n}, \omega\right) \\
& \quad=\frac{\left[1-\epsilon-2 \epsilon \alpha\left(q_{n}, \omega\right)\right] \mathcal{M}_{11}^{(n)}-\left[1+\epsilon+2 \epsilon \alpha\left(q_{n}, \omega\right)\right] \mathcal{M}_{21}^{(n)}}{\left[1-\epsilon-2 \epsilon \alpha\left(q_{n}, \omega\right)\right] \mathcal{M}_{12}^{(n)}-\left[1+\epsilon+2 \epsilon \alpha\left(q_{n}, \omega\right)\right] \mathcal{M}_{22}^{(n)}},
\end{aligned}
$$

where we obtain the elements of the $(2 \times 2)$-matrix $\stackrel{\mathcal{M}}{ }^{(n)}$ by evaluating the product of $L$ transfer matrices $\overleftrightarrow{T}^{(n)}$, whose elements are defined in Eq. (6) for a structure containing $L$ +1 layers. Clearly, in this formalism, the 2D charged layers are coupled through the Coulomb interaction and we assume that there is no interlayer electron hopping between layers. The surface response function in Eq. (7) is a useful tool for calculating the normal-mode spectrum of plasmon excitations for a finite number of layers and can also be employed to investigate the role played by layer separation on the loss function $\operatorname{Im}\left[g\left(q_{n}, \omega\right)\right] .{ }^{14}$ The plasmon dispersion is obtained by setting the denominator in Eq. (7) equal to zero. That is, the plasmon resonances can occur for all values of $n$ and are determined by the angle the in-plane polarization of the in- 
cident electromagnetic field makes with the $x$ axis. In the special case when there is a single layer, we set $\mathcal{M}_{11}^{(n)}$ $=\mathcal{M}_{22}^{(n)}=1$ and $\mathcal{M}_{12}^{(n)}=\mathcal{M}_{21}^{(n)}=0$ in Eq. (7), which becomes ${ }^{15,16}$

$$
g_{\text {single }}\left(q_{n}, \omega\right)=1-\frac{2}{1+\epsilon+2 \epsilon \alpha\left(q_{n}, \omega\right)} .
$$

The surface response function for a semi-infinite slab of dielectric medium can then be deduced from this result by setting the polarization function $\alpha\left(q_{n}, \omega\right)$ equal to zero.

Let us now consider a stationary external point charge $Z^{*} e$ located at $\mathbf{r}_{0}=\left(\mathbf{r}_{\|}=0,-z_{0}\right)$ on the negative side of the polar $z$ axis. The external potential at $\mathbf{r}$ due to the presence of this particle is obtained by solving Poisson's equation $\nabla^{2} \phi_{\text {ext }}(\mathbf{r})$ $=Z^{*} e / \epsilon_{0} \cdot{ }^{17}$ For $-z_{0} \leq z \leq 0$, from the first term of Eq. (1) and derived in the Appendix, we have

$$
\phi_{\text {ext }}(\mathbf{r})=\int \frac{d^{2} \mathbf{q}_{\|}}{(2 \pi)^{2}} \sum_{n=-\infty}^{\infty} e^{-q_{n}\left(z_{0}+z\right)}\left(\frac{Z^{*} e}{2 \epsilon_{0} q_{n}}\right) e^{i q_{x, n} x+i q_{y} y} .
$$

Moreover, from the second term of Eq. (1), vis-a-vis (A4), we find that the induced potential for $z \lesssim 0$ is given by

$$
\phi_{\text {ind }}(\mathbf{r})=-\int \frac{d^{2} \mathbf{q}_{\|}}{(2 \pi)^{2}} \sum_{n=-\infty}^{\infty} e^{-q_{n}\left(z_{0}-z\right)}\left(\frac{Z^{*} e}{2 \epsilon_{0} q_{n}}\right) g\left(q_{n}, 0\right) e^{i q_{x, n} x+i q_{y} y} .
$$

Therefore, the force exerted on the external charge due to the induced charge in the medium is

$$
\mathbf{F}_{\text {ind }}=\left.\frac{\partial}{\partial z} \phi_{\text {ind }}(\mathbf{r})\right|_{z=-z_{0}, \mathbf{r}_{\|}=0} \hat{\mathbf{z}},
$$

which defines the image potential as

$$
\mathcal{U}_{\text {im }}\left(z_{0}\right)=-\frac{\left(Z^{*} e\right)^{2}}{(4 \pi)^{2} \epsilon_{0}} \int d^{2} \mathbf{q}_{\|} \sum_{n=-\infty}^{\infty} e^{-2 q_{n} z_{0}}\left[\frac{g\left(q_{n}, 0\right)}{q_{n}}\right],
$$

where each Fourier component $g\left(q_{n}, 0\right)$ must be calculated as a function of wave vector. Equation (12) is a general formula for the image potential as an integral and a sum over reciprocal-lattice vectors involving the surface response function $g\left(q_{n}, 0\right)$. We can now apply our result to different models of $g$, including graphene. In particular, we use Eq. (12) in a calculation for a layered structure by making use of the results above along with the polarization function for each layer.

For doped graphene, the susceptibility has been calculated at zero temperature $T=0 \mathrm{~K}$ using a low-energy effective Hamiltonian at the $K / K^{\prime}$ point in the Brillouin zone. At zero frequency, we have ${ }^{18-20}$

$$
\begin{aligned}
\operatorname{Re}\left[\chi_{\text {graphene }}^{(0)}\left(q_{\|}, 0\right)\right]= & -\mathcal{D}\left(E_{F}\right)\left\{1-\frac{1}{8 X}\left[2 f_{3}(X) \eta_{+}(X-2)\right.\right. \\
& \left.\left.+\pi X^{2} \eta_{+}(2-X)\right]\right\},
\end{aligned}
$$

where $\eta_{+}(x)$ is the unit step function, $X=q_{\|} / k_{F}, k_{F}$ $=\sqrt{4 \pi n_{2 \mathrm{D}} / g_{s} g_{v}}, n_{2 \mathrm{D}}$ is the sheet density for unmodulated
EGs, $g_{s}$ and $g_{v}$ are the spin and valley degeneracies, the introduced function $f_{3}(X)$ is defined as

$$
f_{3}(X)=2 \sqrt{X^{2}-4}+X^{2} \sin ^{-1}\left(\frac{2}{X}\right),
$$

the density of states at the Fermi energy $E_{F}$ is $\mathcal{D}\left(E_{F}\right)$ $=\sqrt{g_{s} g_{v} n_{2 \mathrm{D}} / \pi} / \hbar v_{F}$, and $v_{F}=1 \times 10^{6} \mathrm{~m} / \mathrm{s}$. For the $2 \mathrm{DEG}$, we have at $T=0 \mathrm{~K}$ (Refs. 21 and 22 )

$$
\begin{aligned}
\operatorname{Re}\left[\chi_{2 \mathrm{D}}^{(0)}\left(q_{\|}, \omega\right)\right] & =\frac{2}{\pi}\left(\frac{m^{*}}{\hbar^{2} q_{\|}}\right)^{2}\left\{\frac{\hbar^{2} q_{\|}^{2}}{2 m^{*}}+\frac{1}{2} \sqrt{\left(\hbar \omega-\frac{\hbar^{2} q_{\|}^{2}}{2 m^{*}}\right)^{2}-\frac{\hbar^{2} q_{\|}^{2}}{m^{*}} E_{F}}\right. \\
& \left.\times \eta_{+}\left[\frac{m^{*}}{\hbar^{2} q_{\|}^{2}}\left(\frac{\hbar^{2} q_{\|}^{2}}{2 m^{*}}-\hbar \omega\right)^{2}-E_{F}\right]+(\omega \rightarrow-\omega)\right\} \\
\approx & -\frac{q_{\|}^{2} n_{2 \mathrm{D}}}{m^{*} \omega^{2}} \text { for } q_{\|} \ll k_{F},
\end{aligned}
$$

where $m^{*}$ is the effective mass of 2DEG, $k_{F}=\sqrt{2 \pi n_{2 \mathrm{D}}}$, and $E_{F}=\hbar^{2} k_{F}^{2} / 2 m^{*}$.

The main difference between Eqs. (13) and (14) arises from the fact that the polarization function for doped graphene has contributions from both the valence and conduction bands at zero temperature. On the other hand, only the energy states in the conduction band contribute to the polarizability of the 2DEG. In addition, for graphene, there is an additional form factor for the overlap of states in the conduction $(s=+1)$ band and valence $(s=-1)$ band given by $F_{s s^{\prime}}\left(\mathbf{k}_{\|}, \mathbf{k}_{\|}^{\prime}\right)=\left(1+s s^{\prime} \cos \theta\right) / 2$ with $\theta$ being the angle between the in-plane wave vectors $\mathbf{k}_{\|}$and $\mathbf{k}_{\|}^{\prime}=\mathbf{q}_{\|}+\mathbf{k}_{\|}$. This leads to both intraband and interband single-particle excitations in two-dimensional graphene whereas only intraband transitions are possible for the 2DEG.

\section{NUMERICAL RESULTS AND DISCUSSION}

In Fig. 1(a), we show plots of the image potential as a function of $k_{F} z_{0}$ for a single layer of 2DEG. In this figure, the effects that the screening produces are shown when a periodic electrostatic potential is applied. Even in the presence of the $1 \mathrm{D}$ periodic potential, the binding energies of the imagepotential states are still given approximately by the hydrogeniclike form of the Rydberg series. Their relative positions with respect to the vacuum level are altered since the image potential with a gated grating is shifted downward relative to its values in the absence of a grating. The ground state has lower energy when a gated grating modulates the 2DEG. The behavior of the image potential for graphene is strikingly similar. Figure 1(b) shows that for a single layer of graphene, $\mathcal{U}_{\text {im }}\left(z_{0}\right)$ continues to behave like $-1 / z_{0}$ for $k_{F} z_{0} \ll 1$ either in the presence or absence of a $1 \mathrm{D}$ periodic modulation.

In Figs. 2(a) and 2(b), we repeated the calculations in Figs. 1(a) and 1(b), respectively, except now there are two parallel layers separated by a distance $a=1000 \AA$. Since interlayer hopping is not included in our model, the layer separation must be chosen sufficiently large to satisfy this condition. As a matter of fact, in graphene, the lattice constant is 

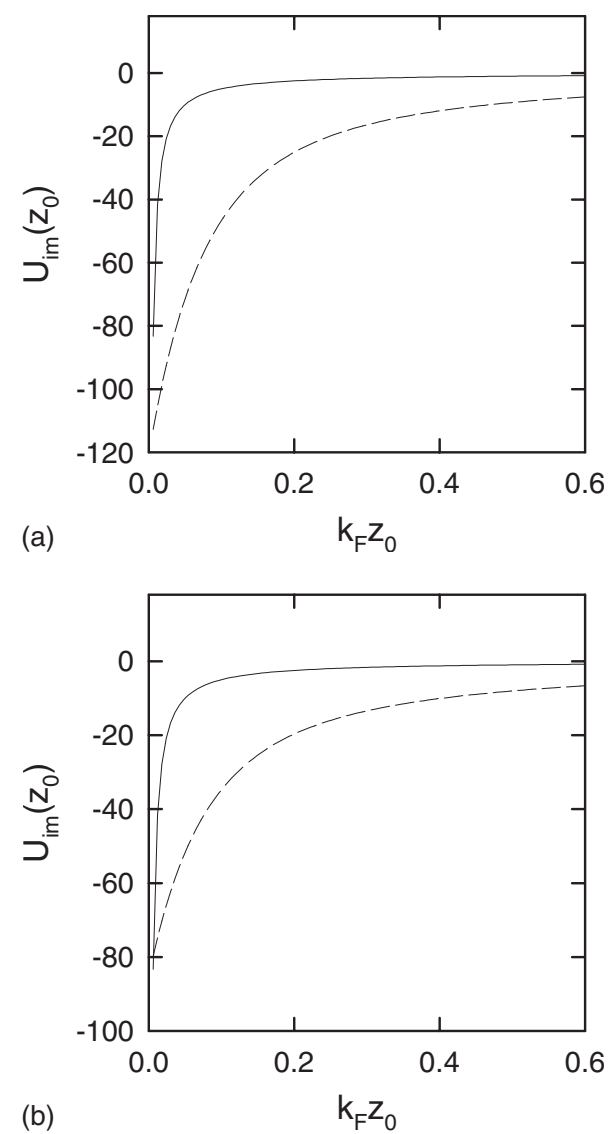

FIG. 1. (a) Plots of the scaled image potential $\mathcal{U}_{\text {im }}$, in units of $\left(Z^{*} e\right)^{2} k_{F} / 8 \pi \epsilon_{0}$, as a function of $k_{F} z_{0}$ for a $2 \mathrm{DEG}$ without a grating (solid curve) and with a gated grating (dashed curve) with period $d=5 \mu \mathrm{m}$. The background dielectric constant is chosen as $\epsilon=13.1$ and the electron effective mass is $m^{*}=0.067 m_{e}$ where $m_{e}$ is the free-electron mass. The 2D electron density is $n_{2 \mathrm{D}}=10^{11} \mathrm{~cm}^{-2}$. (b) The image potential $\mathcal{U}_{\mathrm{im}}$, in units of $\left(Z^{*} e\right)^{2} k_{F} / 8 \pi \epsilon_{0}$, as a function of $k_{F} z_{0}$ for a single layer of graphene in the absence of a grating (solid curve) and with a gated grating present (dashed curve) with period $d=5 \mu \mathrm{m}$. The background dielectric constant is chosen as $\epsilon=5$ and the $2 \mathrm{D}$ electron density is $n_{2 \mathrm{D}}=10^{11} \mathrm{~cm}^{-2}$.

$a_{\text {lattice }}=2.566 \AA$ and $t \approx 2.71 \mathrm{eV}$ is the overlap integral between the nearest carbon atoms. ${ }^{23}$ For this reason, we chose the separation between the graphene layers to satisfy $a$ $\gg a_{\text {lattice }}$ and chose $a=1000 \AA$ in our numerical calculations. Compared with the result in Figs. 1(a) and 1(b) for a single layer, we find a significant increase in the screening effect in a double layer system when $k_{F} z_{0} \ll 1$. Figure 2 (a) shows that for $k_{F} z_{0} \ll 1$ the gated grating sharply increases the magnitude of $\mathcal{U}_{\text {im }}\left(z_{0}\right)$ for a bilayer 2DEG. The screening is the greatest close to the surface but the potential remains negative for all distances $z_{0}$ of the test charge $Z^{*} e$. There is no qualitative difference in the behavior of the image potential for bilayer graphene. Close to the surface layer, the screening of the image potential is stronger when there is no grating compared to the case when a gated grating is present for bilayer graphene.

We have solved the Schrödinger equation for the eigenstates arising from the potential in Fig. 2(b) for bilayer graphene with the gated grating. Setting $Z^{*}=1$, our results
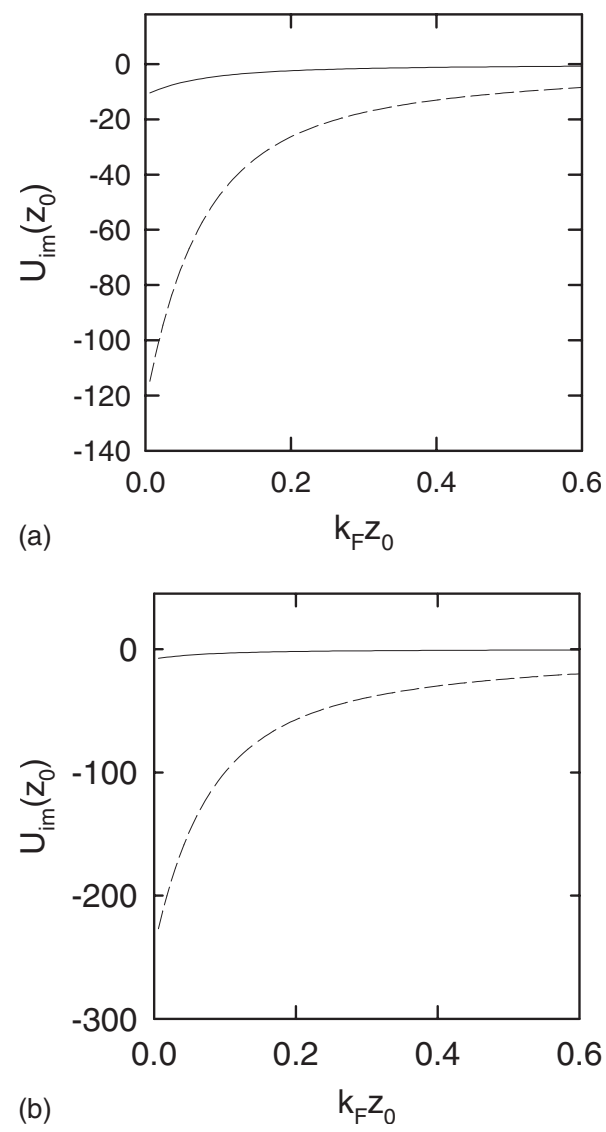

FIG. 2. (a) The image potential $\mathcal{U}_{\text {im }}$, in units of $\left(Z^{*} e\right)^{2} k_{F} / 8 \pi \epsilon_{0}$, as a function of $k_{F} z_{0}$ for a bilayer of 2DEG in the absence of a grating (solid curve) and with a gated grating present (dashed curve). The layer separation is $a=10^{3} \AA$. All other parameters are the same as in Fig. 1(a). (b) The image potential as a function of $k_{F} z_{0}$ for a pair of graphene layers separated by distance $a=10^{3} \AA$ in the absence of a grating (solid curve) and with a gated grating present (dashed curve). All other parameters are the same as in Fig. 1(b).

show that the four lowest eigenvalues for the electron binding energies are $E_{n}=-8.768,-8.053,-7.532$, and $-7.101 \mathrm{eV}$ for $n=1,2,3,4$, respectively. In Fig. 3, we have plotted the wave functions for the three lowest eigenstates in this image potential. These results show that the ground eigenstate (solid curve) has its largest amplitude close to the surface. The spreading of the wave functions (dashed curves) is increased for two excited states. Using the calculated results for the eigenfunctions along with the confining image potential in the Wentzel, Kramers, and Brillouin approximation, we can determine the probability for an electron to tunnel out from its image state.

In Fig. 4, we plot the image potential for a gated grating for three different values of interlayer separation, i.e., $a$ $=100,500$, and $1000 \AA$. The image potential for $a=1000$ $\AA$ differs significantly from the two other curves. The image potential increases in magnitude as $a$ is decreased. However, as we pointed out above, our formalism does not include interlayer hopping and, for this reason, we should keep $a$ $\gg a_{\text {lattice, }}$ compared with the lattice constant.

In general, the effect of the gated grating on a single-layer or bilayer graphene and the $2 \mathrm{DEG}$ is to produce a more 


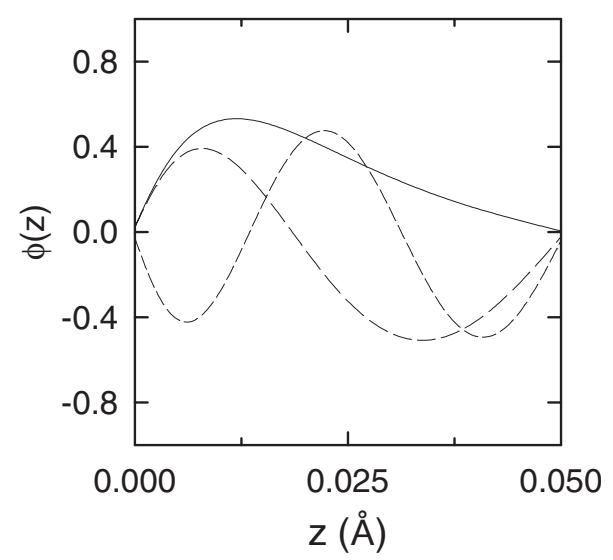

FIG. 3. The wave functions for the ground state (solid curve), the first-excited state (short-dashed curve), and the second-excited state (long-dashed curve) for the image potential with a gated grating present in Fig. 2(b).

attractive image potential. The image potential created near the surface of a $2 \mathrm{DEG}$ or graphene by the induced charge may be manipulated by a gated grating. The way in which the image potential is affected for two layers of 2DES which are coupled via the Coulomb interaction by including tunneling between the layers should be investigated.

We may determine the image plane by using the prescription described in Ref. 4. For each case described above, we can use the image potential in the Schrödinger equation to obtain the energy eigenstates. We can then introduce a $z$-dependent image potential $V_{B}(z)$, with a cutoff, for the surface barrier which we define by $V_{B}(z)=-C / 4 z$ for $z>z_{d}$ and $V_{B}(z)=-C / 4 z_{d}$ for $z \leq z_{d}$. The two arbitrary parameters of this model potential are the depth of the constant potential determined by the parameter $C$ and the width of this region, which defines the distance between the image plane and the surface. ${ }^{24}$ We did not calculate $z_{d}$ in this paper since this is not one of our aims. However, now that we have established a formalism for calculating the image potential, we may proceed to obtain $z_{d}$.

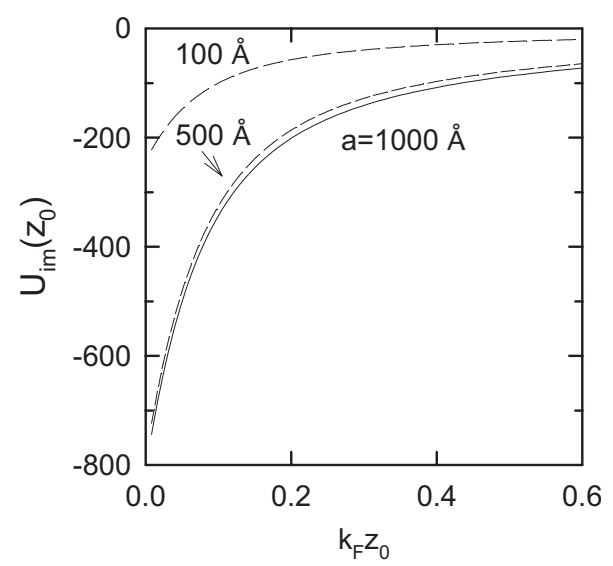

FIG. 4. The image potential $\mathcal{U}_{\text {im }}$, in units of $\left(Z^{*} e\right)^{2} k_{F} / 8 \pi \epsilon_{0}$, as a function of $k_{F} z_{0}$ for bilayer graphene with a gated grating present. The different layer separations in the figure are $a=100,500$, and $1000 \AA$ as indicated. All other parameters are the same as in Fig. $1(\mathrm{~b})$.
We incorporate a conductive grating on top of a conducting sheet for generating and mixing Bragg modes of a reflected/transmitted electromagnetic field. The presence of the Bloch-type modes due to the grating is a direct consequence of the nonlocal mixing of specular and diffraction modes of the reflected electromagnetic field by free-electroninduced optical polarization. In addition, there is interference between a pair of surface optical-polarization waves with opposite Bragg order numbers in the presence of a grating. The interference of these two counterpropagating surface waves leads to the formation of a Wannier-type state with associated electromagnetic fields localized within the gaps of the $e$ grating. These are the effects which contribute to the physical difference between the image potential with and without a periodic grating.

\section{ACKNOWLEDGMENTS}

This work was supported by AFRL under Contract No. FA 9453-07-C-0207 and the (U.S.) Air Force Office of Scientific Research (USAFOSR).

\section{APPENDIX: SURFACE RESPONSE FUNCTION FORMALISM FOR THE MODULATED 2D SYSTEM}

In this appendix, we provide details to show how Eq. (1) is constructed by means of the potentials $\phi_{\text {ext }}$ and $\phi_{\text {ind }}$ in the form of Eqs. (9) and (10). In the region outside the surface, where there is no charge present, the external potential $\phi_{\text {ext }}$ satisfies $\nabla^{2} \phi_{\text {ext }}=0$. Since the system is translationally invariant in the $y$ direction but periodic parallel to the $x$ axis, we have

$$
\phi_{\text {ext }}(\mathbf{r})=\sum_{n=-\infty}^{\infty} \int \frac{d \mathbf{q}_{\|}}{(2 \pi)^{2}} e^{i q_{x, n} x+i q_{y} y} \Phi(z),
$$

where $q_{x, n}=q_{x}+n G$ and $\Phi(z)$ satisfies $d^{2} \Phi / d z^{2}=q_{n}^{2} \Phi$. Consequently,

$$
\phi_{\text {ext }}(\mathbf{r})=\sum_{n=-\infty}^{\infty} \int \frac{d \mathbf{q}_{\|}}{(2 \pi)^{2}} e^{ \pm q_{n} z} e^{i q_{x, n} x+i q_{y} y},
$$

where $q_{n}=\sqrt{q_{x, n}^{2}+q_{y}^{2}}$. Equation (A2) corresponds to the first term in Eq. (1) for the potential outside. We now turn to the second term of Eq. (1).

The induced potential is obtained by solving $\nabla^{2} \phi_{\text {ind }}$ $=\rho_{\text {ind }} / \epsilon_{0}$. This may be rewritten as

$$
\begin{aligned}
\phi_{\text {ind }}(\mathrm{r}, \omega) & =\int d \mathbf{r}^{\prime} v\left(\mathbf{r}, \mathbf{r}^{\prime}\right) \rho_{\text {ind }}\left(\mathbf{r}^{\prime}\right) \\
& =\int d \mathbf{r}^{\prime} d \mathbf{r}^{\prime \prime} v\left(\mathbf{r}, \mathbf{r}^{\prime}\right) \chi^{(0)}\left(\mathbf{r}^{\prime}, \mathbf{r}^{\prime \prime} ; \omega\right) \phi_{\text {ext }}\left(\mathbf{r}^{\prime \prime}\right),
\end{aligned}
$$

where $v\left(\mathbf{r}, \mathbf{r}^{\prime}\right)$ is the Coulomb interaction potential between the electrons in the $2 \mathrm{D}$ system and a point charge $Z^{*} e$, and $\chi^{(0)}\left(\mathbf{r}^{\prime}, \mathbf{r}^{\prime \prime} ; \omega\right)$ is the nonlocal frequency-dependent densitydensity response function. By Fourier transforming the response function and the external potential in the variables $x$ 
and $y$, we obtain after a straightforward calculation the result

$$
\phi_{\text {ind }}(\mathbf{r} ; \omega)=-\sum_{n=-\infty}^{\infty} \int \frac{d \mathbf{q}_{\|}}{(2 \pi)^{2}} g_{n}\left(\mathbf{q}_{\|} ; \omega\right) e^{q_{n}^{z}} e^{i q_{x, n} x+i q_{y} y},
$$

where the Fourier component of the surface response function is defined by

$$
\begin{aligned}
g_{n}\left(\mathbf{q}_{\|} ; \omega\right)= & -\left(\frac{Z^{*} e}{2 \epsilon_{0} q_{n}}\right) \int d z^{\prime} \int d z^{\prime \prime} e^{-q_{n} z^{\prime}} \\
& \times \chi^{(0)}\left(z^{\prime}, z^{\prime \prime} ; q_{x, n}, q_{y} ; \omega\right) \phi_{\text {ext }}\left(z^{\prime \prime}, q_{x, n}, q_{y}\right) .
\end{aligned}
$$

Combining the results in Eqs. (A2) and (A4), we obtain the total potential in Eq. (1).
${ }^{1}$ K. Boger, Th. Fauster, and M. Weinelt, New J. Phys. 7, 110 (2005).

${ }^{2}$ U. Höfer, I. L. Shumay, Ch. Reuß, U. Thomann, W. Wallauer, and Th. Fauster, Science 277, 1480 (1997).

${ }^{3}$ V. Dose, W. Altmann, A. Goldmann, U. Kolac, and J. Rogozik, Phys. Rev. Lett. 52, 1919 (1984).

${ }^{4}$ P. M. Echenique and J. B. Pendry, Prog. Surf. Sci. 32, 111 (1989).

${ }^{5}$ I. R. Collins, P. T. Andrews, and A. R. Law, Phys. Rev. B 38, 13348 (1988).

${ }^{6}$ J. Lehmann, M. Merschdorf, A. Thon, S. Voll, and W. Pfeiffer, Phys. Rev. B 60, 17037 (1999).

${ }^{7}$ Th. Fauster, F. J. Himpsel, J. E. Fischer, and E. W. Plummer, Phys. Rev. Lett. 51, 430 (1983).

${ }^{8}$ P. D. Johnson and N. V. Smith, Phys. Rev. B 27, 2527 (1983).

${ }^{9}$ Brian E. Granger, Petr Král, H. R. Sadeghpour, and Moshe Shapiro, Phys. Rev. Lett. 89, 135506 (2002).

${ }^{10}$ M. Zamkov, N. Woody, B. Shan, H. S. Chakraborty, Z. Chang, U. Thumm, and P. Richard, Phys. Rev. Lett. 93, 156803 (2004).

${ }^{11}$ G. Gumbs, A. Balassis, and P. Fekete, Phys. Rev. B 73, 075411 (2006).
${ }^{12}$ G. Gumbs and D. H. Huang, Phys. Rev. B 75, 115314 (2007).

${ }^{13}$ D. H. Huang, G. Gumbs, P. M. Alsing, and D. A. Cardimona, Phys. Rev. B 77, 165404 (2008).

${ }^{14}$ I. Radović, Lj. Hadžievski, and Z. L. Mišković, Phys. Rev. B 77, 075428 (2008).

${ }^{15}$ A. Eguiluz, T. K. Lee, J. J. Quinn, and K. W. Chiu, Phys. Rev. B 11, 4989 (1975).

${ }^{16}$ B. N. J. Persson, Solid State Commun. 52, 811 (1984).

${ }^{17}$ N. R. Arista, Phys. Rev. A 49, 1885 (1994).

${ }^{18}$ J. Gonzalez, F. Guinea, and M. A. H. Vozmediano, Nucl. Phys. B 424, 595 (1994).

${ }^{19}$ K. W.-K. Shung, Phys. Rev. B 34, 979 (1986).

${ }^{20}$ E. H. Hwang and S. Das Sarma, Phys. Rev. B 75, 205418 (2007).

${ }^{21}$ F. Stern, Phys. Rev. Lett. 18, 546 (1967).

${ }^{22}$ T. Ando, A. B. Fowler, and F. Stern, Rev. Mod. Phys. 54, 437 (1982).

${ }^{23}$ V. Lukose, R. Shankar, and G. Baskaran, Phys. Rev. Lett. 98, 116802 (2007).

${ }^{24}$ P. M. Echenique and J. B. Pendry, J. Phys. C 11, 2065 (1978). 